\title{
Chronic disease prevalence and care among the elderly in urban and rural Beijing, China - a 10/66 Dementia Research Group cross-sectional survey
}

\author{
Zhaorui Liu ${ }^{1}$, Emiliano Albanese ${ }^{2}$, Shuran $\mathrm{Li}^{1}$, Yueqin Huang*1, \\ Cleusa P Ferri' ${ }^{2}$, Fang Yan ${ }^{1}$, Renata Sousa ${ }^{2}$, Weimin Dang ${ }^{1}$ and Martin Prince ${ }^{2}$
}

Address: ${ }^{1}$ Institute of Mental Health, Peking University, Key Laboratory of Mental Health, Ministry of Health (Peking University), No 51 Hua Yuan Bei Road, Haidian District, Beijing 100191, PR China and 2King's College London, Section of Epidemiology, Health Services \& Population Research, De Crespigny Park, London SE5 8AF, UK

Email: Zhaorui Liu - zhaoruiliu@gmail.com; Emiliano Albanese - emiliano.albanese@kcl.ac.uk; Shuran Li - lishuran@bjmu.edu.cn; Yueqin Huang* - dengy@mail.tsinghua.edu.cn; Cleusa P Ferri - cleusa.ferri@kcl.ac.uk; Fang Yan - emillyyf@163.com;

Renata Sousa - renata.sousa@kcl.ac.uk; Weimin Dang - dangweimin@bjmu.edu.cn; Martin Prince - martin.prince@kcl.ac.uk

* Corresponding author

Published: 21 October 2009

BMC Public Health 2009, 9:394 doi:10.1 186/147/-2458-9-394

This article is available from: http://www.biomedcentral.com//47I-2458/9/394

(C) 2009 Liu et al; licensee BioMed Central Ltd.

This is an Open Access article distributed under the terms of the Creative Commons Attribution License (http://creativecommons.org/licenses/by/2.0), which permits unrestricted use, distribution, and reproduction in any medium, provided the original work is properly cited.

\begin{abstract}
Background: Demographic ageing is occurring at an unprecedented rate in China. Chronic diseases and their disabling consequences will become much more common. Public policy has a strong urban bias, and older people living in rural areas may be especially vulnerable due to limited access to good quality healthcare, and low pension coverage. We aim to compare the sociodemographic and health characteristics, health service utilization, needs for care and informal care arrangements of representative samples of older people in two Beijing communities, urban Xicheng and rural Daxing.
\end{abstract}

Methods: A one-phase cross-sectional survey of all those aged 65 years and over was conducted in urban and rural catchment areas in Beijing, China. Assessments included questionnaires, a clinical interview, physical examination, and an informant interview. Prevalence of chronic diseases, self-reported impairments and risk behaviours was calculated adjusting for household clustering. Poisson working models were used to estimate the independent effect of rural versus urban residence, and to explore the predictors of health services utilization.

Results: We interviewed 1002 participants in rural Daxing, and II60 in urban Xicheng. Those in Daxing were more likely to be younger, widowed, less educated, not receiving a pension, and reliant on family transfers. Chronic diseases were more common in Xicheng, when based on self-report rather than clinical assessment. Risk exposures were more common in Daxing. Rural older people were much less likely to access health services, controlling for age and health. Community health services were ineffective, particularly in Daxing, where fewer than $3 \%$ of those with hypertension were adequately controlled. In Daxing, care was provided by family, who had often given up work to do so. In Xicheng, $45 \%$ of those needing care were supported by paid caregivers. Caregiver strain was higher in Xicheng. Dementia was strongly associated with care needs and caregiver strain, but not with medical helpseeking.

Conclusion: Apparent better health in Daxing might be explained by under-diagnosis, under-reporting or selective mortality. Far-reaching structural reforms may be needed to improve access and strengthen rural healthcare. The impact of social and economic change is already apparent in Xicheng, with important implications for future long-term care. 


\section{Background}

China, with over 1.3 billion citizens is the world's most populous country [1]. Demographic ageing is occurring at a rate unprecedented for any world region; the proportion of Chinese aged 65 and over will increase from $4 \%$ in 2000 to $14 \%$ by 2025 , amounting to 200 million older people [2]. The prevention and control of chronic diseases is recognised as an urgent priority in China $[3,4]$. The long term care needs of dependent older people has, comparatively, received much less attention [5-7]. A little over half of China's population lives in rural settings, [8] the proportion among older people is likely to be higher.

The literature on rural/urban differences in health in China suggests three main areas for further study. First, there may be differences in chronic disease prevalence and mortality. Data from the China Health and Nutrition survey showed a 30\% increased mortality for those aged 50 and over in rural compared with urban areas, partly mediated through the lack of amenities and lower wages in rural areas [9]. However, in the third Chinese National Health Service Survey (CNHSS), [10] the prevalence of self-reported physician diagnoses of chronic diseases was lower among rural than urban residents. In the $1991 \mathrm{Bei}-$ jing Longitudinal Study on Aging (BLAS), [11] urban residents were 3.2 times more likely to report chronic disease diagnoses, 4.5 times more likely to report diabetes and 2.5 to three times more likely to report respiratory disease, heart disease and hypertension. On the other hand, levels of disability were similar in the CNHSS, [10] and considerably higher in rural areas in BLAS [11]. Hypertension, when measured directly as opposed to by self-report, is more prevalent in rural areas, [11] and awareness is higher in urban areas [12]. Hence, rural communities seem to be advantaged with respect to some health outcomes, and disadvantaged in others. More research is required to confirm these findings, and to clarify the reasons for the apparent discrepancies.

Second, in China, financing, coverage and access to healthcare depends, largely upon where you live. Only $61 \%$ of rural residents, compared with $82 \%$ of urban dwellers can access health services within one kilometre of their homes [13]. In urban China, there are two employee-based health insurance schemes, one for government and the other for public and private company employees. There is limited cover for dependents, based on a personal annual subscription. Discounts are available for poor people, those with mental disorders, and retirees. In rural China, the government contributes to a common fund which covers healthcare costs but only proportionate to the amount contributed. In 2003, 79\% of rural and $45 \%$ of urban residents did not have meaningful health insurance [12]. Almost $50 \%$ of health care costs are covered by out-of-pockets payments [14] and more than
$35 \%$ of urban and $45 \%$ of rural households cannot afford any health care [15]. The consequences of these disparities for rural and urban older people, in terms of their ability to access healthcare and to manage their chronic diseases, need to be determined.

Third, social protection (encompassing the range of formal and informal mechanisms to provide safety nets and support to poor and disadvantaged members of society) is under threat for older people in China, as its population ages rapidly. In traditional societies social protection is provided by the family and community. With modernisation, these responsibilities are shared by wider society through intergenerational transfers legislated for and managed by the state [16]. However, in the future there will be fewer children available to provide support and care because of the one child policy [17], and social protection by the state, both in terms of pension coverage and insurance against catastrophic healthcare expenditure, remains very patchy [18]. It is important to understand how these processes may be playing out with respect to older people living in urban and rural communities.

In summary, the urban bias of public policy is particularly marked in China, and older people living in rural areas may be especially vulnerable. The Research Agenda on Ageing Project [19] has advocated more research on this group, including demographic and migration patterns; social transitions; family exchanges; health behaviours and use of and access to healthcare. In this paper we seek to pursue this agenda by

a) comparing the socio-demographic and health characteristics of representative samples of older people in two Beijing communities, urban Xicheng and rural Daxing.

b) describing the patterns of recent health service utilization among rural and urban elderly and estimating the independent effect of health conditions, sociodemographic and socioeconomic factors on access to and use of these services.

c) comparing the levels of disability and needs for care, informal care arrangements and extent of carer strain with respect to dependent older people in urban Xicheng and rural Daxing.

\section{Methods}

\section{Study Design and catchment areas}

This study is part of the 10/66 Dementia Research Group's multi-site international programme on dementia and ageing in low and middle income countries. The full study protocol and procedures are available in an open access online publication [20] From January 2004 to April 2005 
we carried out one-phase cross-sectional surveys of all older people (aged 65 years and over) residing in two defined catchment areas: the urban district of Xicheng in the centre of Beijing, close to Tiananmen Square and the 14 villages of Daxing, a rural district 40 kilometres away. In China, rural regions are divided into four classes mainly according to income level, with Class I being the richest and Class IV the poorest [21] - Daxing in common with other rural areas of Beijing is classified under Class I. Participants were identified by means of door-knocking and no exclusion criteria were applied. Sample sizes of 1,000 would allow an estimation of a typical $4.5 \%$ dementia prevalence with a standard error of $1.2 \%$. Community doctors administered the interviews, lasting two to three hours, in participants' homes. Informed consent was obtained from informants and participants. The institutional review boards of the Institute of Psychiatry, King's College London and the Institute of Mental Health, Peking University approved the project.

\section{Measures}

The 10/66 protocol comprises questionnaires on participants' sociodemographic characteristics, health status, risk factor exposures and health service use. A physical examination was carried out and an informant interview administered for information on care arrangements, and caregiver practical, psychological and economic strain. All measures and assessments of the 10/66 protocol are described in detail elsewhere, [20] only those relevant for the purposes of this analysis are described here:

1) Sociodemographic characteristics: gender, age, education, marital status, household living circumstances, sources of income (pension and family support), number of household assets

\section{2) Health Status:}

a. Chronic disease diagnoses established through structured clinical interviewing and or physical examination. Dementia - all those who on the basis of cognitive testing, clinical and informant interview met either or both of the cross-culturally validated 10/66 dementia [22] or DSM IV dementia criteria [23]. Depression - ICD-10 depressive episodes, ascertained using the Geriatric Mental State structured clinical interview [24]. Self-reported stroke, ischemic heart disease and diabetes ("have you ever been told by a doctor that you had a stroke/heart attack/angina/diabetes?"). Chronic obstructive pulmonary disease, defined as reporting a chronic cough with production of sputum for three or more months. Hypertension all those with self-reported hypertension ("have you ever been told by a doctor that you have high blood pressure?") and/or a blood pressure measurement meeting the European Society of Hypertension criteria [25]. (systolic blood pressure $>=140 \mathrm{~mm} \mathrm{Hg}$ and/or diastolic blood pressure $>=95 \mathrm{~mm} \mathrm{Hg}$ ) were considered to have hypertension. Those with self-reported hypertension were considered to be detected. Those with self-reported hypertension but not meeting ESH criteria were considered to be controlled. Those with self-reported treatment ("Were you started on treatment?") were considered to be treated.

b. Physical impairments - self-reported: arthritis or rheumatism; eyesight problems; hearing difficulties or deafness; persistent cough; breathlessness, difficulty breathing or asthma; heart trouble or angina; stomach or intestine problems; faints or blackouts; paralysis, weakness or loss of one leg or arm; skin disorders [26]. Each impairment was rated if it interfered with activities 'a little' or 'a lot'.

c. Disability - measured using the 12 item World Health Organization Disability Assessment Schedule (WHODAS II., developed by WHO for cross-cultural research [27])

d. Risk Factors: Smoking ("ever smoked", "current smoker", "life-time smoking"), hazardous alcohol use ("ever been a heavy drinker" currently and before the age of 60), physical activity ("have you walked at least $0.5 \mathrm{Km}$ in the last month?"), obesity (direct waist circumference in $\mathrm{cm}$ and meets waist circumference criteria for metabolic syndrome)

3) Health Service Utilization: Any use of community services (primary care, hospital outpatient, private doctor, dentistry) and hospital admission during the three months preceding the interview.

4) Dependency (needs for care): we used a series of openended questions addressed to a key informant, to define the family network, to establish if the older person needed and received care from family members or others and to identify who was responsible for organising and providing 'hands on' care. On the basis of these questions, the interviewer coded whether the older person required no care, care for some of the time or care for much of the time.

5) Care arrangements and impact of providing care

a. Informal care arrangements (time spent with the participant, and time spent assisting to communicate, to use transport, to dress, to eat, and for personal hygiene), paid day and night care 
b. The gender of the main carer and their relationship to the cared for older person

c. Carer strain. Carer perceived strain was assessed using the Zarit Burden Interview (ZBI) [28] with 22 items that assess the carer's appraisal of the impact their involvement has had on their lives. The ZBI has been widely used in the USA and Europe, but also in Nigeria and Taiwan $[29,30]$ and in Japan, where it was formally validated [31]. When used in the 10/66 Dementia Research Group pilot studies in 24 centres in Latin America, India, China and Africa the ZBI was found to be practical, culturally relevant, and to have robust psychometric properties [32]. Carer psychological strain was assessed using the 20 item Self-Reporting Questionnaire [33]. Economic strain was assessed according to whether the carer had had to cut back on work to care.

\section{Analysis}

We describe and compare the characteristics of the urban and rural samples according to sociodemographic circumstances ( $\chi^{2}$ and t-test, as appropriate), health status (Poisson regression with robust prevalence ratios controlling for age and gender, and for WHODAS II scores negative binomial regression) and health service utilisation (Poisson regression controlling for age, gender and physical impairments).

1) We compared ( $\chi^{2}$ tests) the proportion of all hypertension cases that are detected, the proportion of detected cases that are a) treated and b) controlled and the proportion of all cases that are controlled.

2) We estimated the independent predictors of health service utilization (accessing any community health service in the last three months) separately for urban and rural samples. Crude and adjusted prevalence ratios (PRs) with 95\% confidence intervals were calculated using Poisson regression adjusting for household clustering, because sometimes two participants of a spouse pair were recruited to the survey. These two persons could not be regarded as statistically independent [34]. Variables included in the model were: dementia diagnosis, one or more physical impairments, age, gender, education, marital status, assets, health insurance and pension.

3) Dementia has previously been shown to be an important determinant of the level and type of informal care, and the extent of carer strain $[35,36]$. Therefore, among those needing care we assessed the effect of dementia diagnosis, and the effect of rural or urban residence (adjusting for age, gender and independently of dementia status) upon: disability (WHO-DAS II) and dependency, time spent assisting with activities of daily living (ADL), carer strain (ZBI scores) and mental health (SRQ-20), and care arrangements (daytime or night time paid carer, cutting back on work to care, additional informal care). To contrast rural and urban samples, we calculated prevalence ratios (PRs) using Poisson regression adjusting for household clustering for dichotomous outcomes, rate ratios using ordinal regression adjusting for household clustering for ordered categorical variables, and mean differences using General Linear Modelling adjusting for household clustering for continuous variables, all with $95 \%$ confidence intervals controlling for age, gender and dementia status.

All analyses were carried out on release 1_5 of the 10/66 dataset using STATA 9.2 (Stata 9.1; Stata Corp., College Station, Texas)

\section{Results}

In all, 2162 (1160 urban and 1002 rural) participants completed the survey, with $95.7 \%$ responding in rural Daxing and $74.3 \%$ in urban Beijing, where more eligible people refused to participate or could not be contacted after at least four attempts. The urban elderly were better educated and older, and less likely to be widowed than their rural counterparts (Table 1). Living alone was unusual in either setting, but urban residents were more likely to be living with their spouse only, and less likely to be living with children. Pension coverage was much lower in the rural $(3.8 \%)$ than the urban sample $(90.5 \%)$. Conversely, family transfers and rental income were much more common in the rural sample. Only nine urban participants and one rural participant reported receiving a disability pension.

All of the self-reported diagnoses and impairments, except hearing problem and limb impairment, were much less common in the rural sample (Table 2). Older people in the rural sample were four times less likely to report three or more limiting impairments and nearly five times more likely to rate their health positively. The picture was different for diagnoses made on the basis of clinical interview and examination. The prevalence of dementia was similar, while that of hypertension was just $20 \%$ lower, and that of uncontrolled hypertension 30\% higher in the rural sample. Significantly more rural $(22.2 \%)$ than urban (13.6\%) elderly reported chronic pain. Rural residents were more likely to have smoked, and to continue to smoke, to have engaged in hazardous drinking, and to be sedentary, but were less likely to be obese.

Rural residents $(6.1 \%)$ were strikingly less likely than urban residents $(38.6 \%)$ to have used any health services over the three months prior to the survey. Underutilisation of services by rural residents was apparent even after controlling for age, gender and number of limiting physi- 
Table I: Social-demographic characteristics in Daxin (rural) and Beijing (urban)

\begin{tabular}{|c|c|c|c|c|c|}
\hline & Urban $(n=1160)$ & Rural $(n=1002)$ & $\chi^{2} / Z$ & $d f$ & $P$ \\
\hline Age group (MV) & 0 & 0 & & & \\
\hline $65-69$ years & $316(27.2 \%)$ & $383(38.2 \%)$ & 40.4 & 3 & $<0.001$ \\
\hline $70-74$ years & $362(31.2 \%)$ & $296(29.5 \%)$ & & & \\
\hline $75-79$ years & $254(21.9 \%)$ & $202(20.2 \%)$ & & & \\
\hline $80+$ years & $228(19.7 \%)$ & $121(12.1 \%)$ & & & \\
\hline Gender (MV) & 0 & 0 & & & \\
\hline Female & $66 \mid(57.0 \%)$ & $556(55.5 \%)$ & 0.5 & 1 & 0.49 \\
\hline Education level (MV) & 0 & 0 & & & \\
\hline No education & $232(20.0 \%)$ & $579(57.8 \%)$ & 491.9 & 3 & $<0.001$ \\
\hline Primary education only & $456(39.3 \%)$ & $373(37.2 \%)$ & & & \\
\hline Completed secondary & $335(28.9 \%)$ & $45(4.5 \%)$ & & & \\
\hline Completed tertiary & $137(|| .8 \%)$ & $5(0.5 \%)$ & & & \\
\hline Marital status (MV) & 0 & 0 & & & \\
\hline Never married & $3(0.3 \%)$ & $22(2.2 \%)$ & 52.0 & 3 & $<0.001$ \\
\hline Married or cohabiting & $829(71.5 \%)$ & $585(58.4 \%)$ & & & \\
\hline Widowed & $326(28.1 \%)$ & $394(39.3 \%)$ & & & \\
\hline Divorced or separated & $2(0.2 \%)$ & $\mathrm{I}(0.1 \%)$ & & & \\
\hline Previous occupation' (MV) & 1 & 1 & & & \\
\hline Professional/Managerial/Clerical & $518(45.0 \%)$ & $37(3.7 \%)$ & 1888.6 & 3 & $<0.001$ \\
\hline Skilled or semi-skilled & $421(36.5 \%)$ & $14(1.4 \%)$ & & & \\
\hline Unskilled & $207(18.0 \%)$ & $13(1.3 \%)$ & & & \\
\hline Agricultural worker & $7(0.6 \%)$ & $938(93.6 \%)$ & & & \\
\hline Living arrangements (MV) & 0 & 0 & & & \\
\hline Alone & $54(4.7 \%)$ & $49(4.9 \%)$ & 202.0 & 3 & $<0.001$ \\
\hline With spouse only & $415(35.8 \%)$ & $194(19.4 \%)$ & & & \\
\hline With children & $446(38.4 \%)$ & $679(67.8 \%)$ & & & \\
\hline With others & $245(21.1 \%)$ & $80(8.0 \%)$ & & & \\
\hline With children under 16 & $217(18.7 \%)$ & $462(46.1 \%)$ & 187.4 & I & $<0.001$ \\
\hline Source of income (MV) & 0 & 0 & & & \\
\hline Government or occupational pension & $1050(90.5 \%)$ & $38(3.8 \%)$ & 1617.5 & I & $<0.001$ \\
\hline Family transfers & $54(4.7 \%)$ & $305(36.4 \%)$ & 347.3 & I & $<0.001$ \\
\hline Disability pension & $9(0.8 \%)$ & $\mathrm{I}(0.1 \%)$ & 5.3 & I & 0.02 \\
\hline Rent & 0 & $122(12.2 \%)$ & 149.7 & I & $<0.001$ \\
\hline Paid work & 0 & $6(0.6 \%)$ & 7.0 & l & 0.008 \\
\hline Have health insurance plan & $14(1.2 \%)$ & $769(76.9 \%)$ & 1327.2 & l & $<0.001$ \\
\hline Number of household assets ${ }^{2}(\mathrm{MV})$ & 0 & 0 & & & \\
\hline $0-3$ assets & $6(0.5 \%)$ & $108(10.8 \%)$ & 113.3 & I & $<0.001$ \\
\hline More than 3 assets & I I54(99.5\%) & $894(89.2 \%)$ & & & \\
\hline
\end{tabular}

$M V=$ Missing values

' Participants were asked 'What was your best (highest level) job?'

2 household assets: television, fridge, water and electricity utilities, telephone, plumbed toilet and plumbed bathroom

cal impairments (Prevalence ratio 0.24, 95\% CI 0.19 to $0.32)$. Underutilisation by rural elderly was equally apparent for primary care services $(3.8 \%$ versus $20.9 \%$, adjusted PR 0.32, 95\% CI 0.23 to 0.45$)$, hospital doctor services (2.2\% versus 23.4\%, adjusted PR 0.14 , 95\% CI 0.09 to $0.22)$ and hospital admission $(0.5 \%$ versus $2.4 \%$, adjusted PR 0.43, 95\% CI 0.15-1.20). Hypertension was less likely to be detected among rural compared with urban residents, and detected cases were much less likely to be controlled (Table 3 ). The net result was that only $2.6 \%$ of all cases of hypertension were controlled in rural Daxing compared with $35.1 \%$ in urban Xicheng.
In both urban and rural sites, numbers of physical impairments were the strongest independent predictors of health service utilisation, after controlling for age, gender, education, assets, pension availability and health insurance (Table 4). Dementia was associated with health service utilization only in rural Daxing, but the association was no longer apparent after controlling for covariates. Economic factors (household assets, receipt of pension and health insurance) predicted health service utilization only in urban Xicheng.

Among the 237 participants who were rated as needing care we described levels of disability and dependency, informal care arrangements and carer strain by site (Table 
Table 2: Health status in Xicheng (urban) and Daxing (rural).

\begin{tabular}{|c|c|c|c|}
\hline Health condition & $\begin{array}{l}\text { Urban, Xicheng } \\
(\mathrm{n}=1160)\end{array}$ & $\begin{array}{l}\text { Rural, Daxing } \\
(\mathrm{n}=1002)\end{array}$ & $\begin{array}{l}\text { Prevalence ratio (rural vs urban) adjusted for } \\
\text { age and gender }\end{array}$ \\
\hline Diagnosed diseases (MV) & 0 & 0 & \\
\hline Dementia & $84(7.2 \%)$ & $56(5.6 \%)$ & $0.96(0.70-1.32)$ \\
\hline History of hypertension, and/or meets ESH criteria & $726(62.6 \%)$ & $500(49.9 \%)$ & $0.80(0.74-0.87)$ \\
\hline Uncontrolled hypertension (ESH criteria) & $47 I(40.6 \%)$ & $487(48.6 \%)$ & $1.29(1.10-1.33)$ \\
\hline ICD-I0 Depression & $3(0.3 \%)$ & $7(0.7 \%)$ & $3.05(0.83-11.2)$ \\
\hline Self-reported diagnoses (MV) & 1 & 0 & \\
\hline Diabetes & $195(16.8 \%)$ & $9(0.9 \%)$ & $0.05(0.03-0.10)$ \\
\hline $\begin{array}{l}\text { Ischaemic heart disease } \\
\text { (myocardial infarction or angina) }\end{array}$ & $115(9.9 \%)$ & $12(1.2 \%)$ & $0.12(0.07-0.23)$ \\
\hline Stroke & $109(9.4 \%)$ & $18(1.8 \%)$ & $0.20(0.12-0.34)$ \\
\hline Chronic obstructive pulmonary disease & $36(3.1 \%)$ & $16(1.6 \%)$ & $0.54(0.30-0.96)$ \\
\hline Self-reported impairments (MV) & 0 & 0 & \\
\hline Arthritis & $165(14.2 \%)$ & $20(2.0 \%)$ & $0.14(0.09-0.23)$ \\
\hline Eye problem & $194(16.7 \%)$ & $65(6.5 \%)$ & $0.4 I(0.3 I-0.55)$ \\
\hline Hearing problem & $142(12.2 \%)$ & $86(8.6 \%)$ & $0.83(0.64-1.08)$ \\
\hline Cough problem & $33(2.8 \%)$ & $14(1.4 \%)$ & $0.5 \mathrm{I}(0.27-0.96)$ \\
\hline Breathing problem & $52(4.5 \%)$ & $19(1.9 \%)$ & $0.45(0.27-0.74)$ \\
\hline Heart problem & $329(28.4 \%)$ & $31(3.1 \%)$ & $0.11(0.08-0.16)$ \\
\hline Gastrointestinal problem & $67(5.8 \%)$ & $12(1.2 \%)$ & $0.22(0.12-0.40)$ \\
\hline Fainting problem & $62(5.3 \%)$ & $10(1.0 \%)$ & $0.19(0.10-0.37)$ \\
\hline Limb problem & $72(6.2 \%)$ & $44(4.4 \%)$ & $0.77(0.53-1.12)$ \\
\hline Skin problem & $12(1.0 \%)$ & $2(0.2 \%)$ & $0.20(0.04-0.94)$ \\
\hline Three or more physical impairments & $208(17.9 \%)$ & $39(3.9 \%)$ & $0.23(0.17-0.33)$ \\
\hline Pain that interferes with life & $158(13.6 \%)$ & $222(22.2 \%)$ & $1.60(1.31-1.94)$ \\
\hline Locomotion (observed) (MV) & 0 & 0 & \\
\hline Obvious abnormality of walking & $53(4.6 \%)$ & $27(2.7 \%)$ & $0.64(0.4 I-1.04)$ \\
\hline WHODAS II disability score (MV) & 10 & 2 & \\
\hline Mean & $8.1 \pm 20.1$ & $8.0 \pm 14.6$ & $\begin{array}{l}1.20(0.96-.49)^{\prime} \\
0.59(0.5 I-0.67)^{2}\end{array}$ \\
\hline Self-rated health (MV) & 0 & 0 & \\
\hline 'Good' or 'very good' & $176(15.2 \%)$ & $690(68.9 \%)$ & $4.59(3.94-5.35)$ \\
\hline Dependency & 0 & 0 & \\
\hline Needs any care & $183(15.8 \%)$ & $54(5.4 \%)$ & $0.4 I(0.30-0.54)$ \\
\hline Needs much care & $119(10.3 \%)$ & $30(3.0 \%)$ & $0.34(0.23-0.51)$ \\
\hline Chronic disease risk factors (MV) & 12 & 4 & \\
\hline Ever smoked & $284(24.5 \%)$ & $336(33.5 \%)$ & $1.28(1.12-1.46)$ \\
\hline Current smoker & $193(16.6 \%)$ & $305(30.4 \%)$ & $1.68(1.43-1.97)$ \\
\hline 20 or more pack years of smoking & $180(15.5 \%)$ & $274(27.3 \%)$ & $1.62(1.38-1.91)$ \\
\hline Hazardous drinker in early life & $26(2.2 \%)$ & $73(7.3 \%)$ & $2.89(1.83-4.52)$ \\
\hline Current hazardous drinker & $17(1.5 \%)$ & $42(4.2 \%)$ & $2.66(1.55-4.56)$ \\
\hline No walks of $>0.5 \mathrm{~km}$ in last month & $209(18.0 \%)$ & $384(38.3 \%)$ & $1.39(1.32-1.47)$ \\
\hline $\begin{array}{l}\text { Obesity } \\
\text { (meets waist circumference criterion for metabolic } \\
\text { syndrome) }\end{array}$ & $530(45.7 \%)$ & $158(15.8 \%)$ & $0.35(0.30-0.41)$ \\
\hline
\end{tabular}

INegative binomial regression

2Zero inflated negative binomial regression

$\mathrm{MV}=$ Missing values

5). In both settings, people with dementia were more disabled than other needing care (mean WHODAS II score 61.1, SD 30.6 versus 33.1, SD 25.7 in Xicheng; 65.5, SD 24.3, $\mathrm{p}<0.001$ versus 33.6, SD 22.7, $\mathrm{p}<0.001$ in Daxing) and more likely to be rated as needing care 'much of the time' (77\% versus 57\%, $\mathrm{p}=0.005$ in Xicheng; $64 \%$ versus $46 \%, \mathrm{p}=0.18$ in Daxing). Carers of people with dementia spent more time assisting with basic activities of daily liv- ing (tests for trend $\chi^{2}=14.1, P=0.001$ in Xicheng, $\chi^{2}=$ 9.9, $P=0.007$ in Daxing), particularly communication, dressing, eating, grooming and toileting. Caregiver strain, measured using the Zarit Burden Interview was also higher among those caring for people with dementia (mean ZBI score 26.4, SD 20.6, $\mathrm{p}<0.001$ versus 12.1, SD 12.6, $\mathrm{p}<0.001$ in Xicheng; 17.1, SD 14.9 versus 5.3, SD 7.7 in Daxing, $\mathrm{p}<0.001)$. It was therefore important to 
Table 3: Detection and control of hypertension, by site

\begin{tabular}{|c|c|c|c|c|c|}
\hline & Urban, Xicheng $(n=1160)$ & Rural, Daxing (n = 1002) & $\chi^{2}$ & $v$ & $P$ \\
\hline Detection and control of hypertension (MV) & 0 & 0 & & & \\
\hline The proportion of all hypertension cases that are detected & $78.5 \%(570 / 726)$ & $50.8 \%(254 / 500)$ & 103.2 & I & $<0.001$ \\
\hline The proportion of detected cases that are treated & $96.8 \%(552 / 570)$ & $99.6 \%(253 / 254)$ & 6.0 & I & 0.015 \\
\hline The proportion of detected cases that are controlled & $44.7 \%(255 / 570)$ & $5.1 \%(13 / 254)$ & 125.7 & I & $<0.001$ \\
\hline The proportion of all cases that are controlled & $35.1 \%(255 / 726)$ & $2.6 \%(13 / 500)$ & 181.5 & I & $<0.001$ \\
\hline
\end{tabular}

$M V=$ Missing values

control for dementia diagnosis, as well as the age and gender of the participant when comparing care-related variables between rural and urban settings (Table 5). Adjusted analyses suggested no differences in levels of disability or dependency between rural and urban older people needing care. However, rural carers spent less time assisting with core activities of daily living, and reported lower levels of strain. Paid care was a common option in urban Beijing; one half of dependent people with dementia and slightly less than one half of all urban dependent people paid for daytime care, with a similar proportion using night time care. Only one rural family used paid daytime care. Instead, rural carers were nearly 12 times more likely to give up or cut back on work to care, and nearly three times more likely to benefit from additional informal care from friends or family.

\section{Discussion}

We carried out a comprehensive one phase survey of two catchment areas in Beijing province; Daxing's rural villages and Xicheng in the heart of Beijing city. There were relatively few non-responders, but the higher proportion in urban Xicheng $(25.7 \%)$ compared with rural Daxing $(4.3 \%)$ creates some potential for response bias. We applied the same catchment area sampling techniques and research protocol in both settings, and the same research group supervised the implementation of the research. Given the proximity, shared language and culture of the two sites, we believe that the comparison was apt and likely to be informative regarding the impact of contrasting infrastructure, policies, lifestyles and family structures on health outcomes and chronic disease care. However, clearly, findings from this comparison cannot be generalised to urban and rural settings in China as a whole. In particular, Daxing is less remote, and better resourced than the majority of rural locations in China. We set out to compare rural and urban samples with respect to the health status of older people, their use of health services, and their needs for informal care. For older people, these three elements are very much interrelated. Other studies that have addressed just one or other of these elements in isolation have not provided a comprehensive overview of chronic diseases, their consequences and their management, and how these might differ in urban and rural populations. However, the broad agenda for this paper has meant that we have not been able to address each topic in detail, for which more indepth dedicated studies will be required.

Self-reported chronic disease diagnoses (diabetes, heart disease and stroke) were more prevalent in urban Xicheng than in rural Daxing. These findings are consistent with

Table 4: Predictors of health service utilization (crude and adjusted robust Prevalence Ratios [PRs] with $95 \%$ confidence intervals $[\mathrm{CI}])$

\begin{tabular}{|c|c|c|c|c|}
\hline & \multicolumn{2}{|c|}{ Crude PRs (95\% Cl) } & \multicolumn{2}{|c|}{ Adjusted PRs* (95\% Cl) } \\
\hline & Urban & Rural & Urban & Rural \\
\hline Dementia & $1.12(0.86-1.45)$ & $2.19(1.05-4.57)$ & $0.94(0.73-1.20)$ & $1.54(0.82-3.06)$ \\
\hline \multicolumn{5}{|l|}{ Number of limiting physical illnesses } \\
\hline None & I (ref) & I (ref) & I(ref) & I (ref) \\
\hline $\mathrm{I}-2$ & $2.32(1.76-2.84)$ & $4.02(2.31-7.00)$ & $2.26(1.79-2.87)$ & $3.82(2.12-6.85)$ \\
\hline 3 or more & $3.78(2.98-4.81)$ & $8.91(4.52-17.6)$ & $3.74(2.94-4.75)$ & $8.31(4.06-17.0)$ \\
\hline Age (per 5 year increment) & $1.03(0.96-1.10)$ & $0.93(0.73-1.17)$ & $1.00(0.96-1.08)$ & $0.80(0.61-1.04)$ \\
\hline Gender (male vs. female) & $0.91(0.79-1.04)$ & $0.87(0.53-I .4 I)$ & $0.89(0.77-1.03)$ & $0.99(0.58-1.70)$ \\
\hline Education (per level) & $1.04(0.99-1.09)$ & $0.90(0.77-1.07)$ & $1.02(0.96-1.08)$ & $0.86(0.63-1.19)$ \\
\hline Assets (per quarter) & $1.28(1.14-1.44)$ & $0.90(0.77-1.05)$ & $1.23(1.09-1.38)$ & $0.84(0.70-1.02)$ \\
\hline Any government or occupational pension & $1.51(1.10-2.08)$ & $1.31(0.43-3.98)$ & $1.46(1.07-1.99)$ & $1.17(0.39-3.57)$ \\
\hline Have health insurance & $1.87(1.33-2.62)$ & $1.75(0.90-3.41)$ & $1.94(1.28-2.95)$ & $1.58(0.82-3.06)$ \\
\hline
\end{tabular}

* Mutually adjusted for all other exposures in the crude model 
Table 5: Levels of disability and dependency, informal care arrangements and carer strain (among those identified as needing care), by site

\begin{tabular}{|c|c|c|c|}
\hline & $\begin{array}{l}\text { Xicheng (urban) } \\
\text { Total } \\
(n=183)\end{array}$ & $\begin{array}{l}\text { Daxing (rural) } \\
\text { Total } \\
(n=54)\end{array}$ & $\begin{array}{l}\text { Effect size for Rural vs. urban contrast, adjusting } \\
\text { for age, gender and dementia status }\end{array}$ \\
\hline $\begin{array}{l}\text { Disability and dependency (in the care } \\
\text { recipient) (MV) }\end{array}$ & 0 & 2 & \\
\hline $\begin{array}{l}\text { WHODAS } 12 \\
\text { Disability score (mean [SD]) }\end{array}$ & $44.2(30.9)$ & $50.2(28.4)$ & $1.7(-6.8,10.3)^{\prime}$ \\
\hline Needs care 'much of the time' & $119(65.0 \%)$ & $30(55.6 \%)$ & $0.8 \mathrm{I}(0.63, \mathrm{I} .05)^{2}$ \\
\hline $\begin{array}{l}\text { Time spent by the carer assisting with } \\
\text { ADL (MV) }\end{array}$ & 0 & 0 & \\
\hline 0 hours & $28(15.3 \%)$ & 7 (13.0\%) & $0.52(0.43-0.63)^{3}$ \\
\hline I-4 hours & $57(31.1 \%)$ & $26(48.1 \%)$ & \\
\hline 5 hours + & $98(53.6 \%)$ & $21(38.9 \%)$ & \\
\hline $\begin{array}{l}\text { Assistance provided for specific ADL (> } \\
\text { one hour/day) (MV) }\end{array}$ & 0 & 0 & \\
\hline Supervision & $16(8.7 \%)$ & $8(14.8 \%)$ & $1.64(0.70-3.85)^{2}$ \\
\hline Communication & $65(35.5 \%)$ & $22(40.7 \%)$ & $1.13(0.78,1.64)^{2}$ \\
\hline Using transport & $8(4.4 \%)$ & $2(3.7 \%)$ & $0.93(0.23,3.75)^{2}$ \\
\hline Dressing & $55(30.1 \%)$ & $10(18.5 \%)$ & $0.59(0.33,1.05)^{2}$ \\
\hline Eating & 57 (31.1\%) & $15(27.8 \%)$ & $0.78(0.50,1.22)^{2}$ \\
\hline Grooming & $57(31.1 \%)$ & $10(18.5 \%)$ & $0.55(0.31,1.00)^{2}$ \\
\hline Toileting & $73(39.9 \%)$ & $14(25.9 \%)$ & $0.6 \mathrm{I}(0.38,0.96)^{2}$ \\
\hline Bathing & $57(31.1 \%)$ & $10(18.5 \%)$ & $0.55(0.31,1.00)^{2}$ \\
\hline Caregiver Strain & 3 & 0 & \\
\hline Zarit Burden Interview Score (mean [SD]) & $17.9(\mid 7.7)$ & $11.4(13.3)$ & $-8.7(-3.9,-13.5)^{\prime}$ \\
\hline \multicolumn{4}{|l|}{ Caregiver mental health } \\
\hline \multicolumn{4}{|l|}{ SRQ-20 Score } \\
\hline Mean (SD) & $0.9(2.3)$ & $1.3(2.6)$ & $0.1(-0.6,0.8)^{\prime}$ \\
\hline Median (IQR & $0(0,1.0)$ & $0(0,1.0)$ & \\
\hline Characteristics of main carer (MV) & 1 & 2 & \\
\hline \multicolumn{4}{|l|}{ Relationship to older person } \\
\hline Spouse & 71 (38.8\%) & $21(38.9 \%)$ & - \\
\hline Child & $69(37.7 \%)$ & $21(38.9 \%)$ & \\
\hline Daughter-/son-in-law or other relative & $13(7.1 \%)$ & II (20.4\%) & \\
\hline Non-relative & $30(16.4 \%)$ & I (1.9\%) & \\
\hline \multicolumn{4}{|l|}{ Gender } \\
\hline Female & $123(67.2 \%)$ & $27(50.0 \%)$ & $1.15(1.05,1.27)^{2}$ \\
\hline Care arrangements (MV) & 0 & 1 & \\
\hline Daytime paid carer & $83(45.4 \%)$ & I (I.9\%) & $0.05(0.01,0.33)^{2}$ \\
\hline Night time paid carer & $81(44.3 \%)$ & 0 & - \\
\hline Carer cut back on work to care & $7(3.8 \%)$ & $26(48.1 \%)$ & $11.7(5.20,26.4)^{2}$ \\
\hline Additional informal care & $13(7.1 \%)$ & $12(22.2 \%)$ & $2.78(1.37,5.63)^{2}$ \\
\hline
\end{tabular}

\footnotetext{
Abbreviations used in the table: MV+ missing values; WHO-DAS $2.0=$ World Health Organization Disability Assessment Schedule (I2-item version); $\mathrm{ADL}=$ activities of daily living; SRQ-20 = Self Reporting Questionnaire (20 items).

IMean difference from a general linear model, with $95 \%$ confidence intervals

2 Prevalence ratios (PRs) from Poisson regression (robust $95 \%$ confidence intervals)

${ }^{3}$ Prevalence ratios (PRs) from ordinal regression,(robust $95 \%$ confidence intervals)
}

reports from previous Chinese surveys, [37-39] but need to be interpreted with caution. There may be systematic under-ascertainment in rural sites because of low levels of awareness and help-seeking, under-detection and undertreatment. Of note, hypertension and dementia, ascertained from clinical assessments in the survey, were similarly prevalent in both sites. Low levels of education may have contributed to ignorance of chronic diseases and under-reporting [11] On the other hand, the prevalence of self-reported impairments was also much lower among older people in rural Daxing, consistent with their better self-rated overall health. Also, when zero inflation was accounted for the disability score count in the rural site was $40 \%$ lower. The lesser needs for care among rural elderly, based upon global assessment by the interviewer, is again consistent with a lower prevalence of chronic disease in Daxing. However, in interpreting these differences in health perception we should bear in mind Amartya 
Sen's allusion to the substantial evidence that "people in states that provide more education and better medical and health facilities are in a better position to diagnose and perceive their own morbidities than the people in less advantaged states, where there is less awareness of treatable conditions (to be distinguished from "natural" states of being)" [40]. Selective mortality may be an additional explanation for the differences in health outcomes. For rural residents a $30 \%$ excess mortality is consistently observed across several data sets, from midlife onwards. The younger age and higher proportion of widows and widowers in Daxing compared with Xicheng is consistent with a difference in midlife mortality between the two populations. Unhealthier lifestyles among the rural elderly may have contributed. Consistent with our findings, a survey in Hubei Province showed higher levels of smoking and alcohol use, and much lower levels of physical activity among older people in rural compared with urban districts [41]. While our data suggests a decline in the prevalence of current smoking among older people compared with the Beijing Longitudinal Ageing Study conducted in $1991 ;[11]$ this decline was more pronounced in urban (from $48.2 \%$ to $16.6 \%$ ) than in rural districts (from $43.5 \%$ to $30.4 \%$ ). In summary, our data, considered in the context of other Chinese surveys, is in no way reassuring regarding the underlying health status of the Chinese rural elderly population.

The differences in our survey in the accessibility and effectiveness of the urban and rural health services were striking. In the Third Chinese National Health Services Survey, rural and urban residents with an illness in the past two weeks were equally likely to seek help from a physician; hospitalisations were less frequent among rural residents, but only among those aged 65 and over $[37,42]$. However, fewer than $7 \%$ of our rural sample as opposed to nearly $40 \%$ of the urban used any health service in the three months preceding the interview. In both sites, physical health was the strongest predictor of the use of health services. Our findings were not explained by the younger age and better health of rural residents. The limited availability of local health services, [38,43] rural poverty, $[37,44]$ the lack of effective insurance cover after the collapse of the rural Cooperative Medical System, and sharp increases in charges under the new fee-for-service system [42] are all likely to be implicated. Economic factors (household assets, receipt of pension and possessing health insurance) were all independently associated with accessing healthcare in urban Xicheng, and may have explained some of the differences in help-seeking between the two sites; limited variance of these factors probably accounts for the lack of association in rural Daxing. Detection and control of hypertension is an important index of the effectiveness of community healthcare. The control of blood-pressure-related disease is a global health priority
[45]. The prevalence of hypertension among older people in China has risen sharply over the period 1991-2006, $[12,46,47]$ and prevention and control are also clear national priorities. Parameters for awareness and control in urban Xicheng were similar to those recently reported for older people in urban Chengdu, [48] while those for rural Daxing were a little worse than those from the national InterASIA survey of 2000-2001, described at that time as 'unacceptably low' [49].

Underutilisation of health services, and lack of routine medical checks may explain the low detection rates $[13,22,50]$. Lack of control among those who were detected and treated was a particular problem in rural Daxing. In Chengdu, [4] lack of control of hypertension was associated with infrequent blood pressure checks, under-treatment, poor treatment adherence, and ignorance of risk factors and potential complications. Hypertension in mid-life is a recognized risk factor for dementia [51-53] Therefore, the extent to which prevention and control of hypertension can be established early in the coming epidemic in China and other LAMIC may have important implications for the size of the predicted increase in numbers of people with dementia in those regions [54]. As others have noted, there is an urgent need to promote access to healthcare in China [42]. Adequate insurance or subsidy to cover health care costs, need to be extended to those outside of the urban cadres, particularly rural residents, those without formal employment and older people [18]. Community healthcare services need to be strengthened. However, attention needs also to be given to increasing the demand for healthcare; health promotion and education to encourage healthy behaviours and help-seeking [55]. Older people need to be targeted [41].

In Daxing, the burden of support and care, where it was required, fell mainly on family members who had often given up work to care. In Xicheng, family members rarely gave up work to care, paid caregivers being employed instead. These stark differences are understandable in the context of China's rapid economic development. Urban Beijing is experiencing a boom, while development in rural areas stagnates. Widening differentials in salary levels between the city and the country drive the trend towards the employment of women from less developed provinces to care for dependent older people in the city. Residential care is costlier, and associated with considerable stigma. Some caution is indicated in interpreting the higher levels of carer strain among urban compared with rural carers, since measurement bias between urban and rural settings may have been implicated; nevertheless, the finding seems plausible. Although the literature is inconsistent on this point, [56] juggling work roles with those of parent, organisational and 'hands-on' caregiver for an 
older relative can be stressful. In Daxing, traditional extended family living arrangements are still the norm, with neighbours and relatives available to provide additional informal care. In China, as in the Dominican Republic [35] and the USA [36] dementia is consistently associated with greater needs for care, more time spent caregiving and greater caregiver strain. Non-communicable diseases are already leading causes of mortality in China [55] and the pace of demographic ageing in China is such that predicted increases in numbers of dependent people [57], and numbers of people with dementia [54] will be greater in absolute and relative terms than for almost any other world region. Developing policies and investing in long-term care should be key priorities, alongside health sector reform.

\section{Conclusion}

Self-reported diabetes, heart disease and stoke were more prevalent in urban Xicheng than in rural Daxing, conversely hypertension and dementia, ascertained from clinical assessments in the survey, were similarly prevalent in both sites. Apparent better health in rural Daxing might be explained by under-diagnosis (and limited access to health care facilities), under-reporting or selective mortality. Care need was common in both sites but whilst informal care was the norm in rural Daxing, paid caregivers were very common in urban Xicheng. The health reform in China should ensure access and long term care in rural settings and at the same time meet the important implications of the socio-cultural and economic changes already apparent in urban China.

\section{Competing interests}

The 10/66 Dementia Research Group works closely with Alzheimer's Disease International (ADI), the non-profit federation of 77 Alzheimer associations around the world. ADI is committed to strengthening Alzheimer associations worldwide, raising awareness regarding dementia and Alzheimer's Disease and advocating for more and better services for people with dementia and their caregivers. ADI is supported in part by grants from GlaxoSmithKline, Novartis, Lundbeck, Pfizer and Eisai.

\section{Authors' contributions}

MP leads the 10/66 Dementia Research Group study and CF acts as research coordinator assisted by EA and RS. SL and $\mathrm{YH}$ are the principal investigators in China, and $\mathrm{ZL}$ is the study coordinator in China. They were assisted in the conduction of the study by FY and WD. ZL and EA wrote the first draft of the paper and carried out the analyses with the assistance of MP and $\mathrm{YH}$. All other authors reviewed the report and provided further contributions and suggestions. All authors approved the final manuscript.

\section{Acknowledgements}

The 10/66 Dementia Research Group study in China has been funded by the World Health Organization (baseline survey) and the Wellcome Trust (GR08002). The authors thank staff in Beijing Xicheng Ping An Hospital and Beijing Daxing Institute of Mental Health Care for assistance with fieldwork and data entry.

\section{References}

I. United Nations Department of Economic and Social Affairs: World Population Prospects: 2006 revision. 2006 [http://esa.un.org/ unpp/].

2. The International Institute for Applied Systems Analysis: China's Population by Age and Sex, 1950-2050. 2008 [http:// www.iiasa.ac.at/collections/llASA Research/SRD/ChinaFood/data/ anim/pop ani.htm].

3. Yang GH: The Transition of Health Mode and the Control Strategy on Chronic Diseases in China. Chinese Journal of Prevention and Control of Chronic Non-communicable diseases 200I, 9: I45-I 48.

4. Zhang PH, Jiao SF, Zhou Y, Li G, Shi Y, Li H, Ren ZY, Wu F, Jiang Y, Guo $X H$, et al.: Studies on prevalence and control of several common chronic diseases among Beijing adults in 2005. Chinese Journal of Epidemiology 2007, 28:625-630.

5. World Health Organization: Towards an International Consensus on Policy for Long-Term Care of the Ageing. Geneva 2000.

6. Prince M, Acosta D, Albanese E, Arizaga R, Ferri C, Guerra M, Huang $Y$, Jacob K, Jimenez-Velazquez IZ, Rodriguez JL, et al.: Ageing and dementia in low and middle income countries - using research to engage with public and policymakers. Int Rev Psychiatry 2008, 20:332-343.

7. Wang L, Yang G, Wang S, Zhou Y: Health service need and utility, and community nursing for the elderly person in urban area. Chinese Journal of Gerontology 2007:1947-1948.

8. The Ministry of Health PRC: Abstract of Health Statistic Report. 2008 [http://www.moh.gov.cn/publicfiles/business/htmlfiles/zwgkzt/ ptjty/200805/3567l.htm].

9. Zimmer Z, Kaneda T, Spess L: An examination of urban versus rural mortality in China using community and individual data. J Gerontol B Psychol Sci Soc Sci 2007, 62:S349-S357.

10. Shi J, Liu M, Zhang Q, Lu M, Quan H: Male and female adult population health status in China: a cross-sectional national survey. BMC Public Health 2008, 8:277.

II. Woo J, Zhang XH, Ho S, Sham A, Tang Z, Fang XH: Influence of different health-care systems on health of older adults: a comparison of Hong Kong, Beijing urban and rural cohorts aged 70 years and older. Australas J Ageing 2008, 27:83-88.

12. Xu L, Wang S, Wang YX, Wang YS, Jonas JB: Prevalence of arterial hypertension in the adult population in rural and urban China: the Beijing eye study. Am J Hypertens 2008, 2 I : I I I 7- I I 23.

13. Centre for Health Statistics and Information-Ministry of Health: An Analysis report of national health services survey in 2003 Beijing - China: Chinese Academy Science \& Peking Union Medcial College; 2004.

14. China National Health Economics Institute: China national health accounts report 2005 Beijing - China: China National Health Economics Institute; 2005.

15. Liu Y, Rao K, Hu SL: People's Republic of China: Toward establishing a rural health protection system. Manila, Asian Development Bank 2002 [http://www.adb.org/Documents/Reports/ PRC Rural Health Protection System/default.asp].

16. Diao L, Tang Z, Sun F: A survey on care need of elderly in Beijing. Chinese Journal of Gerontology 2005, 25:985-986.

17. Li JX: Fertility policy and population ageing in China. Population Research 2000, 24:9-I5.

18. Wagstaff A, Lindelow M, Gao J, Xu L, Qian J: Extending health insurance to the rural population: an impact evaluation of China new cooperative scheme, policy research working paper 4I50. Washington, DC: World Bank; 2007.

19. Andrews G, Clark MJ: The International Year of Older Persons: putting aging and research onto the political agenda. J Gerontol B Psychol Sci Soc Sci 1999, 54:7-10.

20. Prince M, Ferri CP, Acosta D, Albanese E, Arizaga R, Dewey M, Gavrilova SI, Guerra M, Huang Y, Jacob KS, et al.: The protocols for the $10 / 66$ dementia research group population-based research programme. BMC Public Health 2007, 7: 165. 
21. Liu Y, Rao K, Wu J, Gakidou E: China's health system performance. Lancet 2008, 372:1914-1923.

22. Prince M, Acosta D, Chiu H, Scazufca M, Varghese M: Dementia diagnosis in developing countries: a cross-cultural validation study. Lancet 2003, 36 1:909-917.

23. American Psychiatric Association: Diagnostic and Statistical Manual of Mental Disorders, (DSM-IV) Fourth edition. Washington DC: American Psychiatric Association; 1994.

24. Copeland JR, Dewey ME, Griffiths-Jones HM: A computerized psychiatric diagnostic system and case nomenclature for elderly subjects: GMS and AGECAT. Psychol Med 1986, 16:89-99.

25. O'Brien E, Asmar R, Beilin L, Imai Y, Mancia G, Mengden T, Myers M, Padfield P, Palatini P, Parati G, et al.: Practice guidelines of the European Society of Hypertension for clinic, ambulatory and self blood pressure measurement. J Hypertens 2005, 23:697-70I.

26. George LK, Fillenbaum GG: OARS methodology. A decade of experience in geriatric assessment. J Am Geriatr Soc 1985, 33:607-6I5.

27. Rehm J, UTSS: On the development and psychometric testing of the WHO screening instrument to assess disablement in the general population. International Journal of Methods in Psychiatric Research 2000, 8: I I0-I22.

28. Zarit SH, Reever KE, Bach-Peterson J: Relatives of the impaired elderly: correlates of feelings of burden. Gerontologist 1980 20:649-655.

29. Uwakwe R, Modebe I: Disability and care-giving in old age in a Nigerian community. Niger J Clin Pract 2007, 10:58-65.

30. Chou KR, LaMontagne LL, Hepworth JT: Burden experienced by caregivers of relatives with dementia in Taiwan. Nurs Res 1999, 48:206-2|4.

31. Arai Y, Kudo K, Hosokawa T, Washio M, Miura H, Hisamichi S: Reliability and validity of the Japanese version of the Zarit Caregiver Burden interview. Psychiatry Clin Neurosci 1997, $5 \mathrm{I}: 28 \mathrm{I}-287$.

32. 10/66 Dementia Research Group: Care arrangements for people with dementia in developing countries. Int J Geriatr Psychiatry 2004, 19:170-177.

33. Mari JJ, Williams P: A comparison of the validity of two psychiatric screening questionnaires (GHQ- 12 and SRQ-20) in Brazil, using Relative Operating Characteristic (ROC) analysis. Psychol Med 1985, 15:65I-659.

34. Donner A: A regression approach to the analysis of data arising from cluster randomization. Int J Epidemiol 1985 1 4:322-326.

35. Acosta D, Rottbeck R, Rodriguez G, Ferri CP, Prince MJ: The epidemiology of dependency among urban-dwelling older people in the Dominican Republic; a cross-sectional survey. BMC Public Health 2008, 8:285.

36. Ory MG, Hoffman RR III, Yee JL, Tennstedt S, Schulz R: Prevalence and impact of caregiving: a detailed comparison between dementia and nondementia caregivers. Gerontologist 1999, 39:177-185.

37. Feng $X$, Wang D: Medical health service need of Chinese elderly person. Chinese Journal of Health Statistics 1999, 16:287-289.

38. Tang Z, Fang X, Xiang M, Wu X, Diao L, Lin H, Sun F: Research on the health care needs of the elderly in Beijing. Journal of Chinese Hospital Management 2004, 20:464-469.

39. Zhang T, Yang H, Feng W, Zhang X, Guo H, Gong G, Zhang S, Tang $L, X i X$ : Survey on physical health and social support among elderly in two communities in Beijing. Chinese Journal of Epidemiology 2002, 23:240.

40. Sen A: Health: perception versus observation. BMJ 2002, 324:860-86I.

41. Mao Z, Wu B: Urban-rural, age and gender differences in health behaviours in the Chinese population: findings from a survey in Hubei, China. Public Health 2007, I 2 I:761-764.

42. Liu M, Zhang Q, Lu M, Kwon CS, Quan H: Rural and urban disparity in health services utilization in China. Med Care 2007 45:767-774.

43. The Ministry of Health PRC: The Health Statistics Almanac in 2007. 2008 [http://www.moh.gov.cn/publicfiles/business/htmlfiles/ zwgkzt/ptini/200807/37/68.htm].

44. Li Z, Qin H, Guo Q, Cen M: Survey on health status and health care need among urban and rural elderly in Guangxi. Chinese Journal of Gerontology 2007, 27:374-375.
45. MacMahon S, Alderman MH, Lindholm LH, Liu L, Sanchez RA, Seedat YK: Blood-pressure-related disease is a global health priority. Lancet 2008, 37 I: I 480-1482.

46. Wang LD: Comprehensive Report, Chinese nutrition and health survey in 2002 Beijing, China: People's Medical Publishing House; 2005.

47. Department of Disease Control and Prevention Chinese Center for Disease Control: Report on Chronic Diseases in China, Beijing - China 2006.

48. Zhang X, Zhu M, Dib HH, Hu J, Tang S, Zhong T, Ming X: Knowledge, awareness, behavior (KAB) and control of hypertension among urban elderly in Western China. Int J Cardiol 2009, 137:9-15.

49. Gu D, Reynolds K, Wu X, Chen J, Duan X, Muntner P, Huang G, Reynolds RF, Su S, Whelton PK, et al.: Prevalence, awareness, treatment, and control of hypertension in china. Hypertension 2002, 40:920-927.

50. Collaborative research group on the practice guideline on detection evaluation treatment and prevention of hypertension for primary health care doctors: Comparison of the status in control of hypertension between rural and urban community health service centers in Beijing. Chinese Journal of Cardiology 2004, 32: $1021-1025$.

5I. Skoog I, Lernfelt B, Landahl S, Palmertz B, Andreasson LA, Nilsson L, Persson G, Oden A, Svanborg A: I5-year longitudinal study of blood pressure and dementia. Lancet 1996, 347:II4I-I I45.

52. Launer LJ, Masaki K, Petrovitch H, Foley D, Havlik RJ: The association between midlife blood pressure levels and late-life cognitive function. The Honolulu-Asia Aging Study. JAMA 1995 274: 1846-|85|.

53. Launer LJ, Ross GW, Petrovitch H, Masaki K, Foley D, White LR, Havlik RJ: Midlife blood pressure and dementia: the HonoluluAsia aging study. Neurobiol Aging 2000, 2 I:49-55.

54. Ferri CP, Prince M, Brayne C, Brodaty H, Fratiglioni L, Ganguli M, Hall $\mathrm{K}$, Hasegawa K, Hendrie $\mathrm{H}$, Huang $Y$, et al.: Global prevalence of dementia: a Delphi consensus study. Lancet 2005, 366:2112-2117.

55. Yang G, Kong L, Zhao W, Wan X, Zhai Y, Chen LC, Koplan JP: Emergence of chronic non-communicable diseases in China. Lancet 2008, 372:1697-1705.

56. Edwards AB, Zarit SH, Stephens MA, Townsend A: Employed family caregivers of cognitively impaired elderly: an examination of role strain and depressive symptoms. Aging Ment Health 2002, 6:55-61.

57. Harwood RH, Sayer AA, Hirschfeld M: Current and future worldwide prevalence of dependency, its relationship to total population, and dependency ratios. Bull World Health Organ 2004, 82:25I-258.

\section{Pre-publication history}

The pre-publication history for this paper can be accessed here:

http://www.biomedcentral.com/1471-2458/9/394/pre pub

Publish with Bio Med Central and every scientist can read your work free of charge

"BioMed Central will be the most significant development for disseminating the results of biomedical research in our lifetime. "

Sir Paul Nurse, Cancer Research UK

Your research papers will be:

- available free of charge to the entire biomedical community

- peer reviewed and published immediately upon acceptance

- cited in PubMed and archived on PubMed Central

- yours - you keep the copyright
BioMedcentral 